\title{
Slip-Induced Vibration Influences the Grip Reflex: A Pilot Study
}

\author{
Michaël Wiertlewski ${ }^{1,2 *} \quad$ Satoshi Endo ${ }^{3 \dagger} \quad$ Alan M. Wing ${ }^{3 \ddagger} \quad$ Vincent Hayward $^{2 \S}$ \\ ${ }^{1}$ Department of Mechanical Engineering, Northwestern University, Evanston, II, USA \\ 2 UPMC Univ Paris 06, UMR 7222, Institut des Systèmes Intelligents et de Robotique, Paris, France \\ 3 Behavioural Brain Sciences Centre, University of Birmingham, Birmingham, UK
}

\begin{abstract}
Grasping is one of the most common forms of dexterity. So far, most research has focused on slow-varying loads which can be resisted by anticipatory grip adjustments. There are common cases, however, when a rapid, unexpected increase in the load occurs and where the central nervous system must re-adjust the grip dynamically to prevent slippage. During such events, the central nervous system reactively updates the grip force to minimize further escape of an object. While existing theories postulates that the shear strain of the finger pads caused by the load force is a primary source of information for detecting a new load condition, vibrations induced by even minute object slip in the hand might more effectively signal the occurrence of unwanted movement of the object relatively to the hand. With the help of a high-sensitivity force sensor interposed in the load-path of a fast traction-creating device, we recorded the fluctuations of the force projected onto the fingertip when a rapid perturbation was applied to a grasped object. These fluctuations are indicative of slip. The results highlight the existence of a correlation between the amplitude of the vibrations and the grip force modulation, when textural features are present. The study provides promising evidence that the central nervous system exploits vibrations to detect the onset of unwanted movement of an object relatively to the hand to optimally scale the grip force in response to unexpected, rapid load variations.
\end{abstract}

Index Terms: H.5.2 [Information Interfaces and Presentation]: User Interface-Haptic I/O; H.1.2 [Information Systems]: User/Machine Systems-Human information processing; I.3.7

\section{INTRODUCTION}

Control of grip force is crucial for stable object manipulation. In a pinch grasp posture, commonly employed in an experimental setting, the index finger and the thumb laterally compress an object to increase the friction against the contact surface in order to support the object against gravity, as illustrated in Figure 1a. Therefore, by modulating the grip force, the central nervous system controls the friction against the object. Objects we manipulate in everyday situations vary greatly in physical properties. The grip force must be modulated according to changes in the mechanical characteristics of an object and to the frictional properties of the grip surfaces $[14,2]$. For simple, quasi-static loads, this modulation ensures that the lateral grip force against the object surface is maintained at 10 to $40 \%$ over the minimum force necessary to support the object without causing a slip [14, 16,9]. The level of this safety margin may increase with age [4], in the presence of neurological

\footnotetext{
*e-mail: wiertlewski@northwestern.edu

†e-mail: s.endo@bham.ac.uk

†e-mail: a.m.wing@bham.ac.uk

§e-mail: hayward@isir.upmc.fr
}

deficits [11] although the underlying mechanisms could be peripheral (e.g. age-related deficits in innervation) or central (e.g. sensorimotor integration) in origin, or simply due to tribological factors [1]. This safety margin of grip force is considered to prevent accidental slippage in the presence of uncertainty in the motor system, while minimizing fatigue and increasing tactile sensitivity on the fingertips [25]. Furthermore, the central nervous system proactively prevents the object slip across repeated trials of performance by learning the dynamics of the grasping object and building the internal model about that action [8]. If the grip is subject to an unexpected load modification, such as by a collision with other objects, such events may lead to accidental slip. The central nervous system then registers the sensory information and updates the grip force in order to compensate for the perturbation with latency of $60-100 \mathrm{~ms}$ $[15,3,7]$. In the absence of these tactile cues, due to local anesthesia of the digital nerves for instance, the reactive grasping to a load perturbation can be erratic, delayed or even abolished [10, 21]. A typical load force/grip force relationship in the event of object slippage is illustrated in Figure 1b. From a stable value, the tangential loading on the digits increases until it reaches the static friction limit, at which point the object starts sliding. The dynamic friction being lower than the static friction, the maximum admissible load force abruptly decreases as the object starts moving relative to the fingertip. Later, the grip reflex increases the admissible load force until it reaches a point where the object is secured in the hand again. The grip force then slowly returns near to the original safety margin. This reactive grip force is considered to be supraspinal in origin due to the prolonged time span compared to a typical spinal reflex $(\approx 40 \mathrm{~ms})$ and that the neural response to the perturbation can be observed in the sensorimotor cortex at $\approx 60 \mathrm{~ms}$ after the perturbation onset in a EEG study [17]. The motivation behind the present study is to clarify the nature of the haptic events that are important for detection of object slippage, on the basis of which the reactive grip force is programmed. Previous studies suggest that the major haptic source of slip detection involves the cutaneous mechanoreceptors in the fingertips $[13,23]$ and/or in the muscles and joints [19]. The tangential loading on the finger produces strains in the skin and activates the mechanoreceptors which pattern the afferent signals that encode surface information such as shape and texture in a time scale of $100 \mathrm{~ms}$ [13]. In the case of grasping, the state of the digits against the object surface becomes altered with the increasing tangential loading. Initially, the outer part of an ellipse of the surface on the finger pad begins to slide while the inner ellipse remains static. Then, as the force grows, the amount of skin in static contact with the object decreases and eventually fully detaches from the object, leading to slippage [1]. This transition from stick to slip phase is thought to provide cues for the modulation of the grip reflex. Detecting such incipient slip in terms of the ratio of the stick and slip areas and preventing object slippage have been successfully demonstrated in robotic hands [20]. The strain in the fingertips is not the only cue that carries information about incipient slip of the object in the hand, however. For example, the slippage of the object results in vibration of the fingertips. As the grasping digits and the sliding surface develop a relative velocity, asperities 


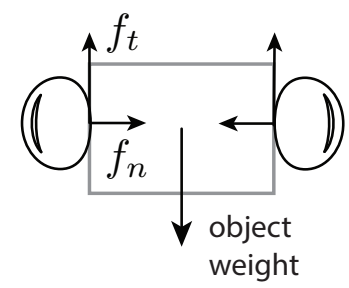

b

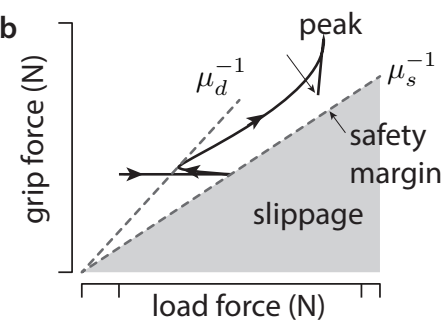

Figure 1: a Posture during pinch grip. $f_{t}$ and $f_{n}$ are the load and grip forces, respectively. $\mathbf{b}$ Evolution of the relationship between grip force and load force before and after slippage. $\mu_{s}$ and $\mu_{d}$ are the static and dynamic coefficients of friction. At the perturbation, the load force increases until it reaches the friction limit and then slippage occurs. The grip force subsequently increases to compensate for the perturbed load force. An overshoot of the grip force ensures the stabilization of the object before reducing the grip force to a safe margin of the static friction limit.

of both surfaces collide, creating high frequency mechanical perturbations [24]. These vibrations travel as mechanical waves in the skin and can be sensed by a large population of the mechanoreceptors [5]. Previously, robotics research has demonstrated that it is possible to detect slippage from the acoustic signature of the transition from the stick to the slip state with the intent to control robotic hands grasp $[12,18]$. Nevertheless, whether the central nervous system uses the vibration for modulating reactive grip force control to a load perturbation has not previously been studied. From these findings, we hypothesize that the central nervous system may use this additional haptic information to program reactive grip force to unintentional slippage. With the help of custom designed apparatus that allows us to capture fine fluctuations of the forces at play, we show that the presence of vibration created by a textured surface is associated with fluctuations in the reactive grip force following a perturbation of the load force.

\section{Methods}

To examine a contribution of vibrotactile cues to in the grip response to an unexpected slip, we measured both the evolution of grip and load forces with high-dynamic range and over a large frequency bandwidth. The object held by the participants, can be fitted with various textured surfaces.

\subsection{Hardware Components}

This experiment used a custom-made apparatus to produce downward force perturbation to the object and to measure the participant's grasping behavior as shown in Figure 2. The participant holds an object equipped with a sensor, which is vertically connected to a capstan system on both ends through a polyethylene cable. The capstan is motorized by two electromagnetic motors (2642 CXR, Faulhaber GmbH, Schonaich, Germany) connected to a drum. An encoder (BTIV 24s 16.24K, Baumer AG, Frauenfeld, Switzerland) serves both as a sensor for the absolute displacement of the object in space, and as a tensioner for the cable. The cable runs to the motor drum, then loops onto the encoder wheel, and continues again on the motors drums, before going to the lower pulley to be connected with the object. The two-loop cable path with the idle wheel provides a symmetric tension to the cable that applied a load using a minimal tension force. The low-tension force provides freedom of motion to the held object while eliminating backlash and reducing friction. The capstan is able to deliver a force up to $20 \mathrm{~N}$ with a static friction not exceeding $0.1 \mathrm{~N}$. The current amplifier (LSC 30/2, 4-Q-DC, Maxon Motor AG, Sachseln, Switzerland) controls the torque applied to the motor, hence the force applied to

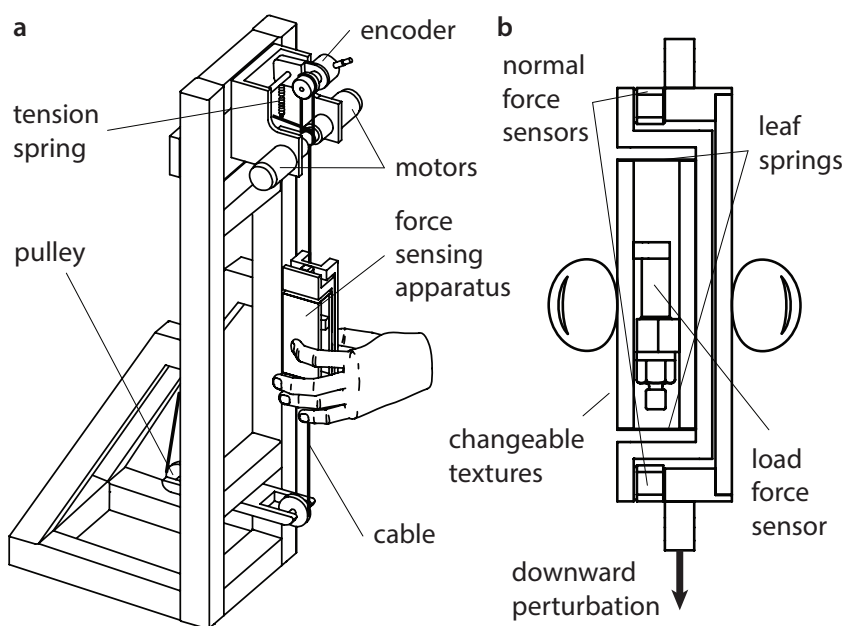

Figure 2: Apparatus used to apply variable load force profiles. a Overview of the experiment. A participant holds the force-sensing apparatus, connected to a capstan. The cable runs around a drum coupled to the motor and to the encoder then on the lower pulley. The encoder is mounted on a spring and tensions the cable. Activating the motor produces a downward force onto the apparatus. b Closer view of the force sensing apparatus. Three high-bandwidth piezoelectric sensors record tangential and normal forces and the position of the contact by calculating the center of pressure. The surface texture of the object can be changed.

the object.The object was fitted with force sensors that measured normal forces and a tangential force. A schematic of the instrument can be seen in Figure 2. Textured surfaces are mounted on both sides of the apparatus. The force sensors measured force exerted on the surface in contact with the index finger as a participant naturally grasped the apparatus. This side of the apparatus is mounted in a cradle suspended by two leaf springs allowing motion in the vertical direction. A high-sensitivity, high stiffness piezoelectric sensor (9217a, Kistler Instrumente AG, Winterthur, Switzerland) is attached to the cradle and measures the force lateral to the fingertip (load force). This sensor is connected to a charge amplifier (5015A, Kistler Instrumente AG, Winterthur, Switzerland). The combination produces baseline noise lower than $10 \mu \mathrm{N}$. The assembly is mounted in another cradle fitted with two force sensors (9313aa1, Kistler Instrumente AG, Winterthur, Switzerland) measuring the force normal to the finger (grip force). These sensors are also connected to a charge amplifier (5073a, Kistler Instrumente AG, Winterthur, Switzerland) and the baseline noise never exceeded $1 \mathrm{mN}$ The frequency response of the entire system was estimated by an impact method and shows a bandwidth of the force measurement that goes up to $500 \mathrm{~Hz}$. These normal forces sensor are placed on both ends of the apparatus, each measuring a fraction of the total normal force applied by the participant. From the ratio of both readings, the position of the center of pressure can be calculated. The position of the contact is estimated from both $\hat{x_{1}}=a \cdot f_{2} / f$ and $\hat{x_{2}}=a .\left(1-f_{1}\right) / f$ where $a$ is the spacing between both sensors, $f_{1}$ and $f_{2}$ are the forces on the upper and lower sensors and $f$ is the total normal force. The variances of the both estimates are then computed from the theoretical noise of the sensor and maximum likelihood integration is performed to compute the final estimate of the position. Calibration has been performed using a motion capture device (Optotrack 3020, NDI International, Waterloo, Canada). The data are acquired by a 16-bit data acquisition board at a sampling frequency of $5 \mathrm{kHz}$. Texture of the sensor apparatus could be changed on each side and the experiment reported here took advantage of this feature. The textures were made out of cast epoxy as de- 
scribed in [24]. One is completely flat yet porous which give a good static friction without much resistance during slippage. The second texture follows a sinusoidal profile with a wavelength of $2 \mathrm{~mm}$ and an amplitude of $50 \mu \mathrm{m}$. The micro-geometry of the overall texture is similar to the flat one. Separately, muscle activity of the first dorsal interosseous was measured using surface EMG (MP150 Biopic Inc., USA) also at 5k Hz sampling frequency. The electrodes were applied with electrolyte gel (Signa Gel, Parker Laboratories, Inc., Orange, NJ, USA) on the skin over the first dorsal interosseus muscle of the right hand. The electrodes were taped to the skin to reduce movement artefacts.

\subsection{Data Analysis}

The EMG data were rectified and then filtered using a zero-lag second-order Butterworth low-pass filter with a cut-off frequency of $36 \mathrm{~Hz}$. The location of the transition between stick and slip was found by locating a maximum in the load force signal during a window of $200 \mathrm{~ms}$ following the perturbation onset. The timing of the reactive grip was registered as the last zero-crossing of the grip force rate before reaching its first peak. The timing of the slip termination was set by the zero-crossing origin of the fingertip and the relative velocity of the finger on the surface, derived from their respective position data. Spectrograms of the load signal were computed using a short-term Fourier transform using a moving Hamming window of $25 \mathrm{~ms}$. The load force was firstly high-pass filtered with a zero-lag second-order Butterworth numerical filter at a cut-off frequency of $20 \mathrm{~Hz}$. Filtering removed the low frequencies to emphasis the vibrotactile component of the signal. Frequency decompositions were computed with a window overlap of $98.5 \%$, every $0.5 \mathrm{~ms}$. The vibration power was calculated from $P_{v}=\frac{1}{T} \int_{t_{1}}^{t_{2}} f_{t}(t)^{2} d t$, where $f_{t}(t)$ is the tangential load force signal, $t_{1}$ is the beginning of slippage, $t_{2}$ time of full stabilization of the load and $T=t_{2}-t_{1}$.

\subsection{Protocol}

A participant sat on a chair in front of the apparatus and held the object using a pinch grasp. The initial gravitational force of the object varied between 1, 2, 3 and $5 \mathrm{~N}$ by adjusting the continuous current in the motors. As the participant lightly held the object, the motor pulled the apparatus downwards at a random time. The sudden increase of the load induced slippage, and subsequent reactive grasp behavior was measured. Perturbation profiles consisted of ramp and impulse of forces in order to prevent the participants forming a stereotypical grip response to the perturbations. The rise of the force was made from the baseline to its final value in $100 \mathrm{~ms}$ and the prescribed additional load force was either $3 \mathrm{~N}$ or $5 \mathrm{~N}$. In the ramp profile, the additional force remained until the end of the trial, whereas the additional force was removed linearly over $100 \mathrm{~ms}$ after its peak to return to the baseline level in the impulse profile. The moisture of the finger was recorded between each trial using a skin moisture measurement device (Moistsense, Moritex Inc, San Jose, CA, USA) as moisture can alter the friction of the finger pads against the object. The experiment consisted of two blocks of 120 trials each, where grasping was performed with one or the other texture surface in each block. The order of the texture presentation was counterbalanced between participants. Within each block, combinations of the baseline and additional force values were repeated 5 times in both ramp and impulse force profiles. The order of these force parameters was completely randomized within each block. In order to reduce the anticipation of the test pull, in randomly selected one third of the trials, no additional force was applied. The experiment took approximately 95 min including 15 min break between the blocks.

\subsection{Participants}

The two participants of this pilot study were right-handed male of age 28 and 29. They washed and dried their hands before the experiment to remove and control extraneous factors that may have affected the friction of the digits such as dirt. Neither participant reported a history of sensory or motor deficit that would have hindered grasp performance. During a trial, a participant comfortably held the object without an excessive level of grip force. The level of the grip force during this baseline period was monitored by the experimenter in real-time. The task of the participant was simply not to drop this object throughout the trial. To prevent damping of the perturbation force using the arm joints, a height-adjustable platform was used for resting the forearm. The participant wore sound insulation headphones (EX-29, DirectSound Headphones 1lc, St. Louis, MO, USA) playing pink noise at a comfortable volume to minimize auditory cues about perturbation. The participants performed the task with the eyes closed to prevent visual feedback.

\section{Results}

The results are presented in two sections. Firstly, we characterize the grasping response to the prescribed various perturbation parameters in group averages of response latency, time and value of the peak grip force. Due to the reactive nature of the behavior investigated in this study, we also calculated the cross-correlation function of the grip force rate to the rate of the force exerted on the object by the motor. The lag of these signals, for which the cross-correlation is at maximum, provides the latency of the grip force modulation to the load of the object and the size of the coefficient indicates the similarity between these two signals at that lag. For statistical comparisons of the group means, a 2 (texture: sinusoid vs. flat) x 2 (perturbation amplitude: $3 \mathrm{~N}$ vs. $5 \mathrm{~N}$ ) x 2 (perturbation type: ramp vs. impulse) ANOVA was performed for each subject. The alpha level was set to 0.05 for reporting statistical significance and only consistently significant results in all participants are reported. Secondly, we focused our analysis on the correlational structure between the vibration generated during the short period of object slippage and the reactive grasp behavior in order to evaluate slip-related haptic cues that are particularly associated with scaling of reactive grip force.

\subsection{Reaction to a load perturbation}

Each trial follows a similar force profile as illustrated in Figure 3. From the onset of the perturbation, the fingertip is first deformed up to a level where the load is higher than the static friction force. When the deformation of the finger reaches the breakaway limit, the state of the object shifts from stick to slip state. This coincides with an impulse of tangential force relief due to the decrease in the resistive force between the contact surface and the fingertips. Following this transition, fingertips slide across the object surface. Collisions between asperities of the surface and the skin generate complex vibration. Approximately $100 \mathrm{~ms}$ after the onset of the perturbation, a participant starts reacting to the sudden increase of the object weight by increasing the grip force to stop object from slipping further. The grip reflex can also be observed from increasing EMG activity of the first dorsal interosseous. After the object is successfully intercepted, the grip force decreases to the near-baseline level prior to the perturbation.

Moisture content. Lower moisture content of the fingertips are mildly correlated with delayed temporal indices of grip performance, including the time of the peak grip force from the onset of the perturbation (Pearson's $r=-0.37, \mathrm{p}<0.005$ ), and the crosscorrelation lag between the grip force rate and the rate of the motor function $(\mathrm{r}=-0.31, \mathrm{p}<0.005)$. The moisture content is also positively correlated with the cross-correlation coefficient, $(\mathrm{r}=0.28, \mathrm{p}$ $<0.005$ ) such that the higher moisture content results in the participants more closely following the dynamics of the object. No other 


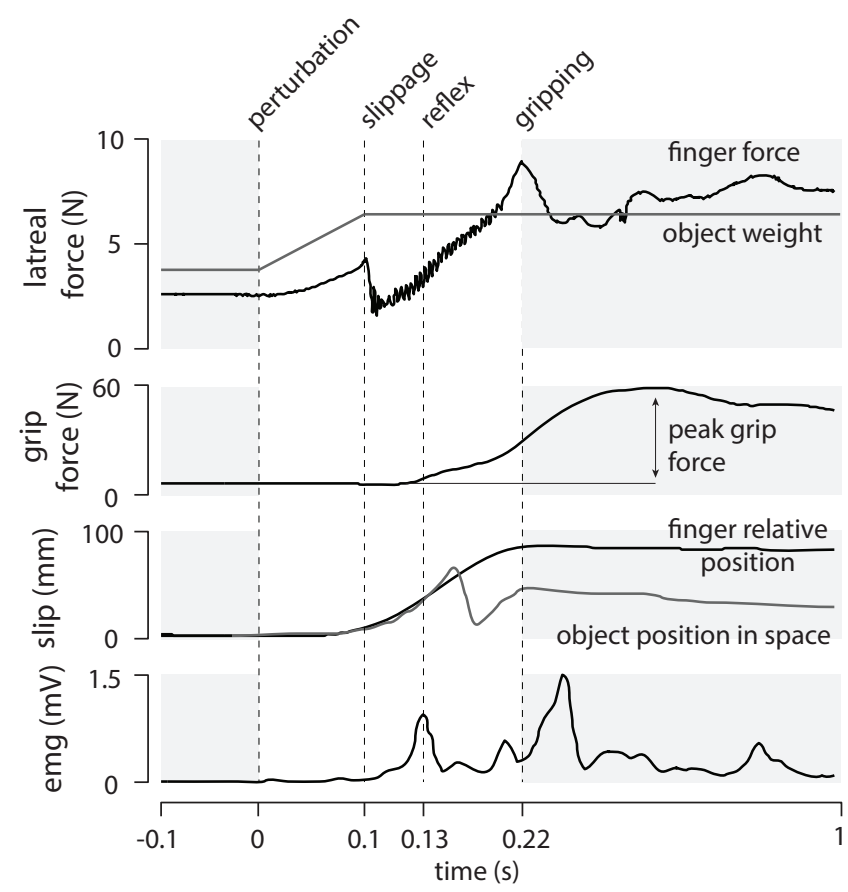

Figure 3: A single-trial example of the grasping and object profiles. The data are time-locked to the onset of the perturbation indicated by the first dotted line. The second dotted line is the initial relief of the tangential force representing the start of the object slippage. The third dotted line shows the peak EMG activity and the forth line indicates the first time at which the slippage was counteracted.

measures are found to be statistically correlated with the moisture content.

Breakaway friction. The maximum load that can sustain the contact before slippage is determined by the static friction coefficient. This friction coefficient is computed from the ratio of load force and grip force, just before the transition from stick to slip. Participant 1 shows a slight effect of the texture on the friction force at breakaway with an average of $0.53 \pm 0.08$ for the flat surface and $0.9 \pm 0.2$ for the sinusoidal surface. The variation between the two surfaces is not as significant for participant 2 where the friction coefficient is $1.2 \pm 0.2$ for the flat surface and $1.08 \pm 0.15$ for the wavy texture.

Slip amplitude. The results concerning the slip amplitude are comparable between the participants, as shown in Figure 4a. The most notable effect is in the perturbation amplitude wherein the larger slip is found for $5 \mathrm{~N}$ perturbation $(\mathrm{p}<0.005)$. Furthermore, there is an overall trend in the ramp force profile causing a larger slip in comparison to the impulse force profile $(\mathrm{p}<0.005)$.

Reactive grip force latency. Overall, the onset of the reactive grip force is faster when the load comprises an abrupt $5 \mathrm{~N}$ increase than $3 \mathrm{~N}$ increase $(\mathrm{p}<0.005)$. Figure $4 \mathrm{~b}$ shows the averages of the grip force latency across the experimental conditions. The averaged latency advantage of $5 \mathrm{~N}$ over $3 \mathrm{~N}$ is $14.22 \mathrm{~ms}$ for the participant 1 and $14.94 \mathrm{~ms}$ for the participant 2. Neither the texture nor the perturbation profile (ramp or impulse shape) has an observable effect on the latency.

Peak grip force. The peak grip force is higher when the object is perturbed with the ramp force profile $(p<0.005)$. Furthermore, there is a main effect of the perturbation amplitude $(p<0.005)$ where the participants execute a higher grip force during $5 \mathrm{~N}$ perturbations. The average of the peak grip force across participants, texture, perturbation force and type can be found in Figure 4c.

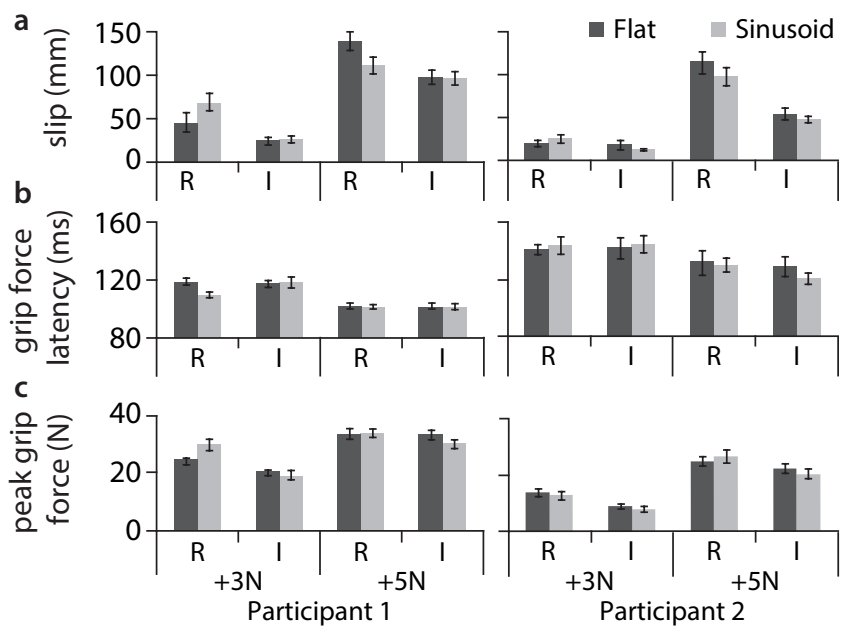

Figure 4: a Change in the slip amplitude, $\mathbf{b}$ reactive grip force latency and $\mathbf{c}$ peak grip force as a function of the grasping surfaces and the perturbation profiles. $\mathrm{R}$ stands for a ramp-and-hold profile and I for an impulsive profile. The error bars represent one standard deviation.

Grip force modulation: cross-correlation lag. The analysis reveals that the grip modulation lags behind the tangential force rate of the object ranging between 155 and $225 \mathrm{~ms}$. A main effect of the perturbation amplitude $(\mathrm{p}<0.005)$ on the lag size indicates that the grip force modulation is delayed further for the $5 \mathrm{~N}$ perturbation $(198.1 \pm 44.5 \mathrm{~ms})$ compared to $3 \mathrm{~N}$ perturbation $(170.3 \pm 40.8$ $\mathrm{ms})$. In addition, an interaction effect between the perturbation amplitude and type was found to be statistically significant $(\mathrm{p}<0.05)$. Namely, the lag is further delayed during the impulse perturbation of $5 \mathrm{~N}$ whereas the perturbation type has little effect during $3 \mathrm{~N}$ perturbation.

Grip force modulation: cross-correlation coefficient. Analysis of the cross-correlation coefficient (Pearson's r) between the grip force rate and tangential force rate applied to the object reveals a main effect for the perturbation amplitude $(p<0.005)$, such that the grip force profile of the participants more closely reflects the perturbation profile when the amplitude is smaller ( $3 \mathrm{~N}: \mathrm{r}=0.74 \pm$ $0.09,3 \mathrm{~N}: \mathrm{r}=0.64 \pm 0.13$ ). The texture is also found to have an effect $(\mathrm{p}<0.05)$ and the correlation is higher when grasping on a sinusoidal surface $(r=0.72 \pm 0.11)$ than on a flat surface $(r=0.66$ \pm 0.12 ).

\subsection{Transient stimulation of the skin}

This section focuses on the analysis of vibration produced by the breakaway and the sliding of the digits on the texture. Figure 5 is a typical example of the signal that is captured by the lateral force sensor. As the object start moving, the index finger experiences an increasing shear stress until the brake away of the object. A sudden decrease in the load force produces a highly energetic impulse shown in the spectrogram of Figure 5. As the index finger continues to slide, vibration is generated by the dynamic interaction between the profile of the contact surface and the skin on the fingertips. In the presence of the perturbation, the velocity of the index finger sliding across the object surface increases, and then it decreases as the object becomes stabilized again. When the object is covered with the sinusoidal texture, the fundamental frequency follows $f=\lambda / v$, where $\lambda$ is the wavelength of the texture, and $v$ is the velocity of the center of contact. The correlation between the velocity and the frequency of the vibration is highlighted in the Figure 5. Three haptic cues are nominated as candidates for slip detection and as parameters for modulating the reactive grip force; 


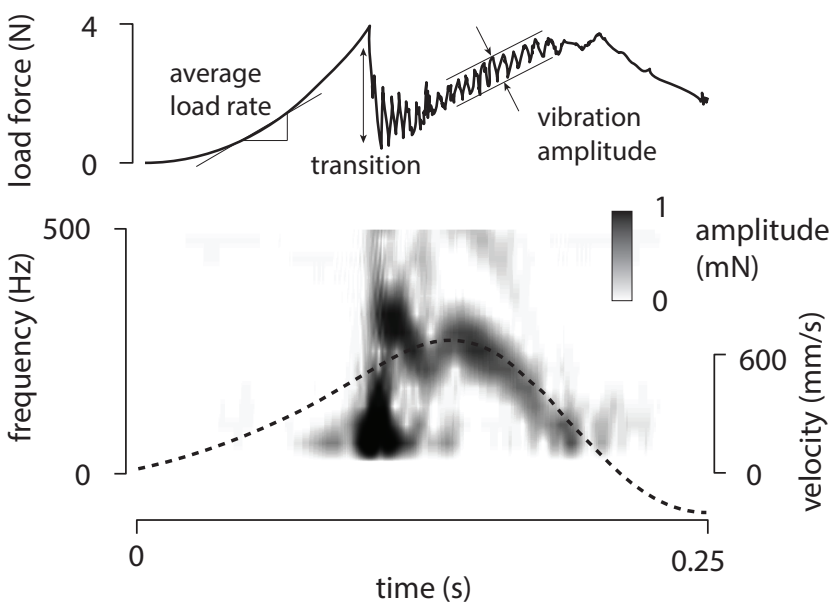

Figure 5: Example of vibration generated when sliding on the sinusoidal textured object. The tangential force (top plot) is subject to frequency-varying vibration after the finger break away from the surface. The time-frequency analysis reveals that the frequency of the vibration is modulated by the velocity of the index finger moving across the object surface (dashed line).

the accumulated load force before commencing the slip phase (stick to slip transition), the peak force rate of this accumulation (friction force rate), and the vibration-related power spectral density in the load force (vibration power). Figure 6 illustrates the changes in the peak grip force with respect to variances of these three haptic cues in scatter plots. In particular, a moderate to high correlation with the peak grip force is observed in friction force rate for both flat and sinusoidal textured objects. In contrast, a linear trend of the vibration power with respect to the peak grip force is observed for the object with the sinusoidal texture but not with the flat texture. No reliable correlation is found for the stick to slip transition. The linear regression coefficients $(\alpha)$ and offsets $(\beta)$ for each pair of variables and their correlation coefficients $(r)$ are reported in Table 1 . To confirm the relative contributions of these haptic cues in predicting subsequent grip force modulation, a stepwise multiple regression analysis was carried out. The statistical procedure estimated that there is a large overlap in the variances of the friction force rate and that of the vibration power, and the contribution of the vibration power is negligible on the flat surface performance (Participant $1 ; \mathrm{p}=.12$, Participant $2 ; \mathrm{p}=.75)$ in the presence of the friction force rate $(\mathrm{p}<$ $0.005)$. Conversely, when participants performed the grasping on the sinusoidal texture, the vibration power became a more reliable predictor $(\mathrm{p}<0.005)$, while the contribution of the friction force rate was rejected from the regression model (Participant $1 ; p=.74$, Participant 2; $\mathrm{p}=.15$ ).

Table 1: Linear regression coefficients of the peak grip force

\begin{tabular}{ll|cc|cc|} 
& & \multicolumn{2}{|c}{ participant 1 } & \multicolumn{2}{c}{ participant 2 } \\
& & flat & sine & flat & sine \\
\hline \multirow{2}{*}{ friction force rate } & $\alpha$ & 1.4 & 1.2 & 1.1 & 0.71 \\
(N/s) & $\beta$ & 0.9 & -5.2 & 11.1 & 16.1 \\
& $r$ & 0.52 & 0.57 & 0.57 & 0.33 \\
\hline \multirow{2}{*}{ stick to slip tran- } & $\alpha$ & 4.22 & 4 & 3.1 & 2 \\
sition (N) & $\beta$ & 15.6 & 25.2 & 24.7 & 25.1 \\
& $r$ & 0.25 & 0.17 & 0.22 & 0.01 \\
\hline \multirow{2}{*}{ vibration power } & $\alpha$ & 0.018 & 0.09 & 0.023 & 0.088 \\
(a.u) & $\beta$ & 14.6 & 19.6 & 30.8 & 24.3 \\
& $r$ & 0.06 & 0.68 & 0.14 & 0.44
\end{tabular}

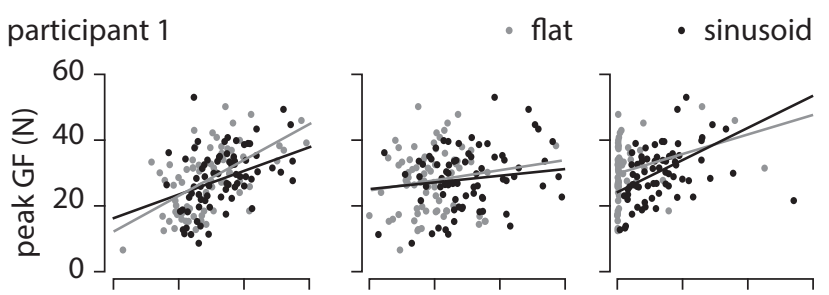

$$
\text { participant } 2
$$
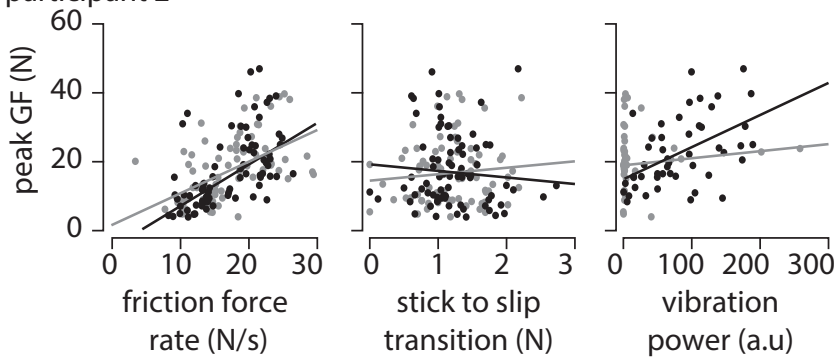

Figure 6: Scatter plots of the peak grip force change as a function of the load force rate, the force drop during transition between stick and slip states and the power of vibration created by sliding on the textured surface. Data includes both perturbation amplitude conditions.

\section{Discussion}

The present study provides an insight into how a vibratory cue generated by an accidental slip of an object may be used to perceive the state of the object and to efficiently terminate the slippage by executing appropriate reactive grip force. While the initial strain rate of the finger pads was consistently associated with variances in amplitude of the reactive grip force, our study shows that the vibratory power caused by the sliding of an object also plays a role as a haptic cue for perceiving its motion. This effect is present when the texture creates a high level of vibration during slippage. On the one hand, no effect of the texture on the amplitude of the peak grip force or the temporal indices of the grasping responses was found and both textures exhibited a similar friction. On the other hand, the vibratory characteristics generated from sliding over these two textures are considerably different, strengthening the argument that the vibration played an important role in the change of the grip modulation in this study. The regression model further indicates that the vibration explains some of the variance in the peak grip force. These results lead us to postulate that the central nervous system takes advantage of vibration features to modulate the generation of additional grip force. The analysis of the vibration is in accord with the finding of $[24,5]$ which demonstrated that the temporal frequency of the vibration is proportional to the velocity of the finger swiping across the contact surface. This is believed to be caused by the contacting skin rapidly compressing and releasing as it complies with the texture of the object, creating vibration in the $100-400 \mathrm{~Hz}$ bandwidth. In the case of re-grasping a sliding object, the mechanoreceptors are passively stimulated. It has been shown that the velocity estimation of periodic and non-periodic surfaces are strongly biased by the spatial frequency of the texture [6]. During rapid events, such as the one that occurs during an accidental object slippage, the central nervous system has to resolve the velocity of the moving object as quickly as possible and the vibration of the texture is likely to be a strong and more reliable cue in this circumstance. At the same time, the vibration power evolves with the square root of the relative velocity (assuming constant friction force) between the fingertip and the object [24]. Therefore, the vibratory power delivered by the sliding of both bodies is likely to provide an efficient haptic cue for the estimation of relative velocity. The Pacinian channels are often recognized to code the envelope of 
vibration and are ideal candidate to provide such a cue for the modulation of reactive grip force to a slipping object. As suggested in previous studies, the initial reflex is likely to be triggered by the increase of shear force built on the finger prior to the object slippage as shown by the strong correlation of this parameter to the peak grip force. However, we believe that vibration plays an important role in modulating reactive grip force after the object begins to slip as the vibratory characteristic between the finger pad and contact surface could dilute slip-related information in the fingertip topography.

\section{Conclusion}

We devised a mechanical setup that allowed us to measure the grip and the load forces that arise from holding an object in precision grip. The apparatus was fitted with actuators that could apply a controlled perturbation to the object and produce involuntary slippage of the object. The measurements extracted from a series of sudden increases of load force gave us a unique insight into the vibrotactile cues that are related to the reactive grip modulation. The analysis of the force data, along with the displacement of the fingertip on the object surface, showed a strong influence of the surface texture in contact with the fingertip on the peak grip force exerted by our participants. These results not only further our understandings of reactive grasp behavior to haptic events, but also they provide useful information for designing robotic systems or training dexterity skills such as for rehabilitating grasping in post-stroke patients. Another application of this work would be in conjunction with virtual environments where the vibrotactile signal can be used as an input of vibrotactile actuators to improve the user's perception of object slippage. Future work will focus on replicating these results with more participants. A larger sample of population will provide us with more generalized view of various parameters influencing the grasp behavior such as the fingertip moisture. The device is being used to study other aspects of the influence of vibration on grip reflex [22]. Furthermore, the results reported in this paper uses a correlational method between vibration power and the modulation of the grip force to a perturbation of the load in order to demonstrate their relationships. Our aim is to directly manipulate the vibration frequency of the object by mounting a voice-coil that can generate vibration as the object slides in the hand. Such study would give us more direct causal evidence for the role of vibration in reactive grip force modulation.

\section{ACKNOWLEDGEMENTS}

The authors wish to thank Mark Farrington for input on the platform. This work has been supported by the Technical Committee on Haptics, Student Exchange Program. MW and VH acknowledge the support of European Research Council, Advanced Grant PATCH, agreement No. 247300 and SE and AW the European Union Seventh Framework Programme grant agreement No. 287888 (CogLaboration) and No. 228844 (NanoBioTouch).

\section{REFERENCES}

[1] T. André, V. Lévesque, V. Hayward, P. Lefèvre, and J. Thonnard. Effect of skin hydration on the dynamics of fingertip gripping contact. Journal of The Royal Society Interface, 8(64):1574-1583, 2011.

[2] G. Cadoret and A. Smith. Friction, not texture, dictates grip forces used during object manipulation. Journal of Neurophysiology, 75(5):1963-1969, 1996.

[3] K. Cole and J. Abbs. Grip force adjustments evoked by load force perturbations of a grasped object. Journal of neurophysiology, 60(4):1513-1522, 1988.

[4] K. Cole, D. Rotella, and J. Harper. Mechanisms for age-related changes of fingertip forces during precision gripping and lifting in adults. The Journal of neuroscience, 19(8):3238-3247, 1999.

[5] B. Delhaye, V. Hayward, P. Lefèvre, and J. Thonnard. Textureinduced vibrations in the forearm during tactile exploration. Frontiers in Behavioral Neuroscience, 6, 2012.
[6] A. Dépeault, E. Meftah, and C. Chapman. Tactile speed scaling: contributions of time and space. Journal of neurophysiology, 99(3):14221434, 2008.

[7] C. Dugas and A. Smith. Responses of cerebellar purkinje cells to slip of a hand-held object. Journal of neurophysiology, 67(3):483-495, 1992.

[8] J. Flanagan and A. Wing. The role of internal models in motion planning and control: evidence from grip force adjustments during movements of hand-held loads. The Journal of Neuroscience, 17(4):1519$1528,1997$.

[9] A. Goodwin, P. Jenmalm, and R. Johansson. Control of grip force when tilting objects: effect of curvature of grasped surfaces and applied tangential torque. The Journal of neuroscience, 18(24):1072410734, 1998.

[10] C. Häger-Ross and R. Johansson. Nondigital afferent input in reactive control of fingertip forces during precision grip. Experimental brain research, 110(1):131-141, 1996.

[11] J. Hermsdörfer, E. Hagl, D. Nowak, and C. Marquardt. Grip force control during object manipulation in cerebral stroke. Clinical Neurophysiology, 114(5):915-929, 2003.

[12] R. Howe and M. Cutkosky. Sensing skin acceleration for slip and texture perception. In Robotics and Automation, 1989. Proceedings., 1989 IEEE International Conference on, pages 145-150. IEEE, 1989.

[13] R. Johansson and I. Birznieks. First spikes in ensembles of human tactile afferents code complex spatial fingertip events. Nature neuroscience, 7(2):170-177, 2004.

[14] R. Johansson and G. Westling. Roles of glabrous skin receptors and sensorimotor memory in automatic control of precision grip when lifting rougher or more slippery objects. Experimental Brain Research, 56(3):550-564, 1984.

[15] R. Johansson and G. Westling. Signals in tactile afferents from the fingers eliciting adaptive motor responses during precision grip. Experimental Brain Research, 66(1):141-154, 1987.

[16] H. Kinoshita, L. Bäckström, J. Flanagan, and R. Johansson. Tangential torque effects on the control of grip forces when holding objects with a precision grip. Journal of Neurophysiology, 78(3):1619-1630, 1997.

[17] D. Kourtis, H. Kwok, N. Roach, A. Wing, and P. Praamstra. Maintaining grip: anticipatory and reactive eeg responses to load perturbations. Journal of neurophysiology, 99(2):545-553, 2008.

[18] P. J. Kyberd and P. H. Chappell. Characterization of an optical and acoustic touch and slip sensor for autonomous manipulation. Measurement Science and Technology, 3(10):969, 1992.

[19] V. Macefield, C. Häger-Ross, and R. Johansson. Control of grip force during restraint of an object held between finger and thumb: responses of cutaneous afferents from the digits. Experimental Brain Research, 108(1):155-171, 1996.

[20] T. Maeno, S. Hiromitsu, and T. Kawai. Control of grasping force by detecting stick/slip distribution at the curved surface of an elastic finger. In Robotics and Automation. Proceedings. ICRA'OO. IEEE International Conference on, volume 4, pages 3895-3900. IEEE, 2000.

[21] D. Nowak, J. Hermsdörfer, S. Glasauer, J. Philipp, L. Meyer, and N. Mai. The effects of digital anaesthesia on predictive grip force adjustments during vertical movements of a grasped object. European Journal of Neuroscience, 14(4):756-762, 2001.

[22] S. Okamoto, M. Wiertlewski, and V. Hayward. Anticipatory vibrotactile cueing facilitates grip force adjustment. In World Haptics Conference (WHC),. IEEE, 2013.

[23] H. Wheat, L. Salo, and A. Goodwin. Human ability to scale and discriminate forces typical of those occurring during grasp and manipulation. The Journal of neuroscience, 24(13):3394-3401, 2004.

[24] M. Wiertlewski, C. Hudin, and V. Hayward. On the 1/f noise and noninteger harmonic decay of the interaction of a finger sliding on flat and sinusoidal surfaces. In World Haptics Conference (WHC), pages 25-30. IEEE, 2011.

[25] V. Zatsiorsky, F. Gao, and M. Latash. Motor control goes beyond physics: differential effects of gravity and inertia on finger forces during manipulation of hand-held objects. Experimental brain research, 162(3):300-308, 2005. 